\title{
Symbolic Quantitative Robustness Analysis of Timed Automata ${ }^{\star}$
}

\author{
Ocan Sankur \\ Université Libre de Bruxelles, Brussels, Belgium
}

\begin{abstract}
We study the robust safety problem for timed automata under guard imprecisions which consists in computing an imprecision parameter under which a safety specification holds. We give a symbolic semi-algorithm for the problem based on a parametric data structure, and evaluate its performance in comparison with a recently published one, and with a binary search on enlargement values.
\end{abstract}

\section{Introduction}

Timed automata [2] are a well-established formal model for real-time systems. They can be used to model systems as finite automata, while using, in addition, a finite number of clocks to impose timing constraints on the transitions. Efficient model checking algorithms have been developed and implemented in tools such as Uppaal [6], IF [12. Timed automata are, however, abstract models, and therefore make idealistic assumptions on timings, such as perfect continuity of clocks, infinite-precision time measures and instantaneous reaction times.

As for any abstract formalism, once desired properties of a system are proven on the model, a crucial question that remains is the robustness of these properties against the assumptions that have been made. What is the extent to which the assumptions behind the model can be relaxed while a given property still holds?

In this work, we are interested in the robustness against timing imprecisions. An important amount of work has been done in the timed automata literature to endow timed automata with a realistic semantics, and take imprecisions into account, e.g. 18151]. The works [24] and [14] showed that perturbations on clocks, i.e. imprecisions or clock drifts, regardless of how small they are, may drastically change the behavior in some models. These observations mean that there is a need for verification tools to check the robustness of timed automata, that is, whether the behavior of a given timed automaton is preserved in the presence of perturbations, and to compute safe bounds on such perturbations.

We consider the robustness of timed automata for safety properties under timing imprecisions modeled by guard enlargement, consisting in relaxing each guard of the form $x \in[a, b]$ to $x \in[a-\delta, b+\delta]$ where $\delta$ is a parameter. Our goal is to decide if for some $\delta>0$, the enlarged timed automaton satisfies its specification (Problem 1), and if this is the case, compute a safe upper bound

\footnotetext{
* Supported by the ERC starting grant inVEST (FP7-279499).
} 
on $\delta$ (Problem 2). We insist on the importance of both problems: while the first one decides the robustness of the model, the second one quantifies it by actually giving a bound under which the model is correct. This would allow one for instance to choose an appropriate hardware to implement the model [151].

Background. The formulation of Problem 1 has been studied starting with 2414 for safety properties, and extended to LTL and richer specifications, e.g. 910] using region-based techniques which cannot be applied efficiently. A symbolic zone-based algorithm was given in [13. for flat timed automata, that is, without nested cycles by applying acceleration on its cycles. Problem 2 has been answered in 20] for flat timed automata, where the given algorithm computes the largest upper bound on $\delta$ satisfying the specification. The flatness is a rather restrictive hypothesis since, for instance, it is easily violated when the system is obtained by composition of timed automata that contain cycles. Recently, a zone-based algorithm and a tool to solve Problem 1 for general timed automata was given [21]; but the algorithm does not compute any bound on $\delta$. The latter algorithm is based, roughly, on extending the standard forward exploration of the state space augmented with the acceleration of all cycles encountered during the search, with some tricks to optimize the computations. In 22, refinements between interfaces are studied in a game-based framework including syntactic enlargement to account for imprecisions. In [25 26] the authors use the fact that replacing all guards by closed ones allow one to verify finite paths (and the case of a periodic external synchronization) but this does not help in the analysis of the accumulation of imprecisions, nor can it allow one to compute a bound on $\delta$.

Results. In this paper, we present a symbolic procedure to simultaneously solve Problems 1 and 2 for general timed automata; if the given model is robust, a safe upper bound on $\delta$ (which may not be the largest one) is output. The procedure is a semi-algorithm since we do not know whether it terminates although it did terminate on most of our experiments. It consists in a state-space exploration with an efficient parametric data structure which treats the enlargement $\delta$ as an unknown parameter, combined with an acceleration procedure for some of the cycles. We do not systematically accelerate cycles, but rather adopt a "lazy" approach: during the exploration, when the accumulated imprecisions go beyond a threshold, we accelerate some cycles that may be responsible for this accumulation. This greatly reduces the computation overhead compared to a systematic acceleration. We also adapt several abstraction operations such as LU abstraction [5], and closure inclusion [19] to the parametric setting to reduce the state space. We ran experiments to evaluate the performance of our procedure. Compared to 21, ours terminated faster in most cases, and sometimes with several orders of magnitude. To truly evaluate the gain of a parametric analysis, we also compared with a binary search on the values of $\delta$ using an exact model checker. Our procedure was often faster except against a low precision binary search (i.e. with few iterations). Section 6 contains a more detailed discussion. 


\section{Definitions}

Given a finite set of clock $\mathcal{C}$, we call valuations the elements of $\mathbb{R}_{>0}^{\mathcal{C}}$. For $R \subseteq \mathcal{C}$ and a valuation $v, v[R \leftarrow 0]$ is the valuation defined by $v[R \leftarrow 0](x)=v(x)$ for $x \in \mathcal{C} \backslash R$ and $v[R \leftarrow 0](x)=0$ for $x \in R$. Given $d \in \mathbb{R}_{\geq 0}$ and a valuation $v, v+d$ is defined by $(v+d)(x)=v(x)+d$ for all $x \in \mathcal{C}$. We extend these operations to sets of valuations in the obvious way. We write $\mathbf{0}$ for the valuation that assigns 0 to every clock. An atomic guard is a formula of the form $k \leq x$ or $x \leq l$ where $x, y \in \mathcal{C}, k, l \in \mathbb{Q}$. A guard is a conjunction of atomic guards. A valuation $v$ satisfies a guard $g$, denoted $v \models g$, if all atomic guards are satisfied when each $x \in \mathcal{C}$ is replaced by $v(x)$. We write $\Phi_{\mathcal{C}}$ for the set of guards built on $\mathcal{C}$.

A timed automaton $\mathcal{A}$ is a tuple $\left(\mathcal{L}, \operatorname{Inv}, \ell_{0}, \mathcal{C}, E\right)$, where $\mathcal{L}$ is a finite set of locations, Inv : $\mathcal{L} \rightarrow \Phi_{\mathcal{C}}$ the invariants, $\mathcal{C}$ is a finite set of clocks, $E \subseteq \mathcal{L} \times \Phi_{\mathcal{C}} \times$ $2^{\mathcal{C}} \times \mathcal{L}$ is a set of edges, and $\ell_{0} \in \mathcal{L}$ is the initial location. An edge $e=\left(\ell, g, R, \ell^{\prime}\right)$ is also written as $\ell \stackrel{g, R}{\longrightarrow} \ell^{\prime}$. For any location $\ell$, let $E(\ell)$ denote the set of edges leaving $\ell$. Following the literature on robustness in timed automata (e.g. 24[14]) we only consider timed automata with closed and rectangular guards (that is, we do not allow constraints of the form $x-y \leq k$ ). We also assume that all invariants contain an upper bound for all clocks.

A run of $\mathcal{A}$ is a sequence $q_{1} e_{1} q_{2} e_{2} \ldots q_{n}$ where $q_{i} \in \mathcal{L} \times \mathbb{R}_{\geq 0}^{\mathcal{C}}$, and writing $q_{i}=(\ell, v)$, we have $v \in \operatorname{Inv}(\ell)$, and either $e_{i} \in \mathbb{R}_{>0}$, in which case $\bar{q}_{i+1}=\left(\ell, v+e_{i}\right)$, or $e_{i}=\left(\ell, g, R, \ell^{\prime}\right) \in E$, in which case $v \models g$ and $q_{i+1}=\left(\ell^{\prime}, v[R \leftarrow 0]\right)$. We say that the run $r$ is along $e_{1} e_{2} \ldots e_{n-1}$. A path is a sequence of edges whose endpoint locations are equal. Given a path $\rho=e_{1} e_{2} \ldots e_{n-1}$ and states $q, q^{\prime}$, we write $q \stackrel{\rho}{\rightarrow} q^{\prime}$ if there is a run from $q$ to $q^{\prime}$ along $e_{1} e_{2} \ldots e_{n-1}$. We write $q \Rightarrow q^{\prime}$ if there is a path $\rho$ with $q \stackrel{\rho}{\rightarrow} q^{\prime}$. We also note $q \stackrel{\rho^{+}}{\rightarrow} q^{\prime}$ if there is a run from $q$ to $q^{\prime}$ along an arbitrary (positive) number of repetitions of $\rho$. A cycle of a timed automaton is a path that ends in the location it starts. As in 2414, we assume that all cycles of considered timed automata reset all clocks at least once. Such cycles are called progress cycles.

Regions. The first decidability results on timed automata relied on a finite partition of the state space to so called regions, which can be defined using simple constraints on clocks [2]. We say that $\frac{1}{\eta}$ is the granularity of a timed automaton $\mathcal{A}$, if $\eta$ is the smallest integer such that all constants of $\mathcal{A}$ are multiples of $\frac{1}{\eta}$. We generalize the definition of regions to arbitrary granularities. Let us denote $\mathbb{N}_{\eta}=\frac{1}{\eta} \mathbb{N}$. Consider a timed automaton $\mathcal{A}$, with granularity $\frac{1}{\eta}$, and consider a bound function $\alpha: \mathcal{C} \rightarrow \mathbb{N}_{\eta}$ mapping each clock to a bound. An $\alpha, \eta$-region is defined by choosing

- for each clock $x \in \mathcal{C}$, a constraint among $\left\{x=k \mid k \in \mathbb{N}_{\eta}, k \leq \alpha(x)\right\} \cup\{k-$ $\left.\frac{1}{\eta}<x<k \mid k \in \mathbb{N}_{\eta}, \frac{1}{\eta} \leq k \leq \alpha(x)\right\} \cup\{x>\alpha(x)\}$.

- for each pair $x, y \in \mathcal{C}$ for which we chose the constraints $k-\frac{1}{\eta}<x<k$, and $l-\frac{1}{\eta}<y<l$, choose one constraint among $\operatorname{frac}(x)<\operatorname{frac}(y), \operatorname{frac}(x)=$ $\operatorname{frac}(y), \operatorname{or} \operatorname{frac}(x)>\operatorname{frac}(y)$, where $\operatorname{frac}(\cdot)$ denotes the fractional part. 
It can be shown that $\alpha, \eta$-regions finitely partition the state space $\mathbb{R}^{\mathcal{C}}$. For $\eta=$ 1 , this is the usual definition of regions. Given timed automata with rational constants, one often rescales the constants to work with integers. In the context of enlargement, however, it will be more convenient to work directly with rationals.

Difference-Bound Matrices. Because the number of regions is exponential in the input size, the region-based algorithms are not practical. Symbolic algorithms were rather given as an efficient solution based on zones which are convex subsets of the state space definable by clock constraints. Formally, a zone $Z$ is a convex subset of $\mathbb{R}^{\mathcal{C}}$ definable by a conjunction of constraints of the form $x \leq k, l \leq x$, and $x-y \leq m$ where $x, y \in \mathcal{C}, k, l \in \mathbb{Q}>0$ and $m \in \mathbb{Q}$. Note that because all guards of the timed automata we consider are closed, all zones that appear during a state-space exploration are closed. Hence, we do not need to distinguish strict and non-strict inequalities as done for general timed automata.

We recall a few basic operations defined on zones. Let Post $>0(Z)$ denote the zone describing the time-successors of $Z$, i.e., Post $\operatorname{Po}_{\geq 0}(Z)=\left\{v \in \mathbb{R}_{\geq 0}^{C} \mid \exists t \geq\right.$ $0, v-t \in Z\}$; and similarly $\operatorname{Pre}_{\geq 0}(Z)=\left\{v \in \mathbb{R}_{\geq 0}^{C} \mid \exists t \geq 0, v+t \in Z\right\}$. Given $R \subseteq$ $\mathcal{C}$, we let $\operatorname{Reset}_{R}(Z)$ be the zone $\left\{v \in \mathbb{R}_{\geq 0}^{C} \mid \exists v^{\prime} \in Z, v=v^{\prime}[R \leftarrow 0]\right\}$, and Free $_{R}(Z)=\left\{v \in \mathbb{R}_{\geq 0}^{C} \mid \exists v^{\prime} \in Z, v^{\prime}=v[R \leftarrow 0]\right\}$. Intersection is denoted $Z \cap Z^{\prime}$. Zones can be represented by difference-bound matrices (DBM) which are $\left|\mathcal{C}_{0}\right| \times\left|\mathcal{C}_{0}\right|$-matrices with values in $\mathbb{Q}\left[16\right.$. Let us define $\mathcal{C}_{0}=\mathcal{C} \cup\{0\}$, where 0 is seen as a clock whose value is always 0 . Intuitively, each component $(x, y) \in \mathcal{C}_{0}^{2}$ of a DBM stores a bound on the difference $x-y$. For any DBM $M$, let $\llbracket M \rrbracket$ denote the zone it defines. DBMs admit reduced forms (a.k.a. normal form), and successor computation can be done efficiently (in $O\left(|\mathcal{C}|^{3}\right)$ ). We refer the reader to 7] for details. All of the above operations can be computed with DBMs. By a slight abuse of notation, we will use the same operations for DBMs as for zones, for instance, we will write $M^{\prime}=$ Post $_{\geq 0}(M)$ where $M$ and $M^{\prime}$ are reduced DBMs such that $\llbracket M^{\prime} \rrbracket=$ Post $_{\geq 0} \llbracket M \rrbracket$. We define an extended zone as a pair $(\ell, Z)$ where $\ell$ is a location and $Z$ a zone. Given an edge $e=\left(\ell, g, R, \ell^{\prime}\right)$, and an extended zone $(\ell, Z)$, we define $\operatorname{Post}_{e}((\ell, Z))=\operatorname{Inv}\left(\ell^{\prime}\right) \cap \operatorname{Post}_{\geq 0}\left(g \cap \operatorname{Reset}_{R}(Z)\right)$, and $\operatorname{Pre}_{e}((\ell, Z))=\operatorname{Pre}_{\geq 0}\left(g \cap\right.$ Free $\left._{R}\left(\operatorname{Inv}\left(\ell^{\prime}\right) \cap Z\right)\right)$. For a path $\rho=e_{1} e_{2} \ldots e_{n}$, we define $\operatorname{Post}_{\rho}$ and $\operatorname{Pre}_{\rho}$ by iteratively applying Post $_{e_{i}}$ and $\mathrm{Pre}_{e_{i}}$ respectively.

Enlargement. We model timing imprecisions in timed automata by the enlargements of the guards and invariants of by rational values $\nu>0$. The enlargement of an atomic guard $k \leq x$ (resp. $x \leq l)$ is denoted $(k \leq x)_{\nu}=k-\nu \leq x$ (resp. $\left.(x \leq l)_{\nu}=x \leq l+\nu\right)$. The enlargement of a guard $g$, denoted $(g)_{\nu}$ is obtained by enlarging all its conjuncts. We denote by $\mathcal{A}_{\nu}$ the timed automaton obtained from $\mathcal{A}$ by enlarging all its guards and invariants by $\nu$.

If $\nu$ is known, one could analyze $\mathcal{A}_{\nu}$ with known techniques, since this is still a timed automaton (with a possibly different granularity). Here, we are rather interesting in a parametric analysis. We thus consider a symbolic parameter $\delta$. The parametric enlargement of a guard $g$, denoted $(g)_{\delta}$ is defined by replacing $\nu$ by the symbol $\delta$ in the above definition. We will always denote rational enlargement 


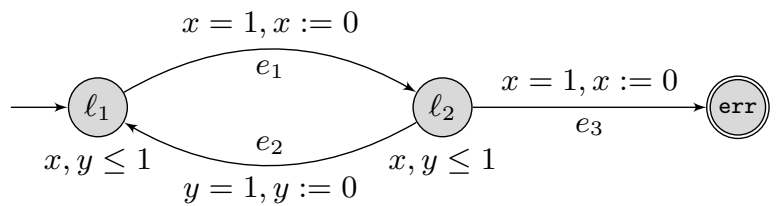

Fig. 1. A timed automaton representing the two processes $P_{1}$ and $P_{2}$ instantiated with period $p=1$, and a buffer size of 1 . The guard under the locations are the invariants. The edge $e_{1}$ represents the arrival of a token in the buffer (period of 1) while $e_{2}$ represents process $P_{2}$ reading a token from the buffer. The error state is reached via $e_{3}$ if two tokens are pushed to the buffer without any read in between. Without enlargement, any reachable state at location $\ell_{2}$ satisfies $x=0 \wedge y=1$, so the error state is not reachable. Under enlargement by $\nu=\frac{1}{10}$, after the first transition, location $\ell_{2}$ is reached by the set of states $1-\nu \leq y \leq 1+\nu \wedge 0 \leq x \leq 2 \nu$ due to the enlargement of the guards and invariants. A simple calculation shows that the set of reachable states at location $\ell_{2}$ after $k$ cycles is $1-(2 k+1) \nu \leq y \leq 1+\nu \wedge 0 \leq x \leq 2 k \nu \wedge 1-(2 k+1) \nu \leq y-x \leq 1+\nu$. Thus, for $k=5$, we get $y \leq 1+\nu \wedge x \leq 1 \wedge-\nu \leq y-x \leq 1+\nu$, and $x=1 \wedge y=1$ is in this set, from which the error state is reachable.

values by $\nu$, and the parameter symbol by $\delta$. Similarly, parametrically enlarged timed automata are denoted $\mathcal{A}_{\delta}$. For $\nu>0$, the instantiation of a parametrically enlarged guard $(g)_{\delta}$ by $\nu$ is denoted $(g)_{\delta}[\delta \leftarrow \nu]$ which is $(g)_{\nu}$.

Accumulation of Imprecisions. In some timed automata even the smallest enlargement can lead to drastically different behaviors due to the accumulation of the imprecisions over long runs [24]. As an example, consider the following simple problem. Two processes $P_{1}, P_{2}$ execute on different machines and communicate via a finite buffer. Every $p$ time units, Process $P_{1}$ finishes a computation and pushes a token to the buffer; while $P_{2}$ reads a token from the buffer with the same period. We assume $P_{2}$ has an offset of $p$. The buffer will clearly not overflow in this system. However, assuming the slightest delay in the execution of $P_{2}$, or the slightest decrease in the execution time of $P_{1}$ leads to a buffer overflow since the delays will accumulate indefinitely. Figure 1 represents this system.

\section{Accelerating Cycles}

The original robust reachability algorithm of 2414] consists in an exploration of the region graph, augmented with the addition of the images of all cycles neighboring reachable states. The idea is that when the guards are enlarged, these neighboring cycles become reachable, and they precisely capture all states that become reachable in the timed automaton for all values of $\delta$. Thus, this algorithm computes the set $\cap_{\nu>0} \operatorname{Reach}\left(\mathcal{A}_{\nu}\right)$, where Reach $\left(\mathcal{A}_{\nu}\right)$ denotes the states that are reachable in $\mathcal{A}_{\nu}$. A symbolic algorithm for this problem was given in [13] for flat timed automata, i.e. without nested cycles, and later improved in [20].

In this section, we summarize some of these results from [2414 1320] that we use in the rest of the paper. Let us fix a timed automaton $\left(\mathcal{L}, \ln v, \ell_{0}, \mathcal{C}, E\right)$, and 
a cycle $\rho$. The following lemma shows the effect of repeating cycles in enlarged timed automata, formalizing our observations on Fig. 1.

Lemma 1 ([20]). Consider any extended zone $(\ell, Z)$, and a progress cycle $\rho$ of $\mathcal{A}$ that starts in $\ell$. If $\operatorname{Pre}_{\rho}^{*}(\top) \cap Z \neq \emptyset$, then starting from any state of $\operatorname{Pre}_{\rho}^{*}(\top) \cap$ $Z$, for any $\nu>0$, all states of $\operatorname{Post}_{(\rho)_{\nu}}^{*}(\top)$ are reachable in $\mathcal{A}_{\nu}$, by repeating $\rho$.

As an example, consider Fig. 1. For the cycle $\rho=e_{2} e_{1}$ that starts at $\ell_{2}$, we have $\operatorname{Pre}_{\rho}^{*}(\top)=x, y \leq 1 \wedge x-y \leq 0$, and $\operatorname{Post}_{(\rho)_{\nu}}^{*}(\top)=x, y \leq 1+\nu \wedge x-y \leq 0$. Since the point $(0,1)$ is reachable and belongs to Pre ${ }_{\rho}^{*}$, all states of $\operatorname{Post}_{(\rho)_{\nu}}^{*}(\top)$ are reachable, and in particular $(1,1)$ from which the error state is reachable.

It is known that the above lemma does not hold for non-progress cycles; nevertheless, it was shown that in this case, $\operatorname{Post}_{(\rho)_{\nu}}^{*}(\top)$ is an over-approximation of the states reachable by repeating $\rho$ under enlargement [11. Thus, the algorithm of 2414] may have false negatives (may answer "not robust" even though it is) but not false positives on timed automata with arbitrary cycles.

Conversely, it has been shown that any state that belongs to $\cap_{\nu>0} \operatorname{Reach}\left(\mathcal{A}_{\nu}\right)$ is reachable along an alternation of exact runs and repetitions of enlarged cycles.

Lemma 2 ([14]). Assume that $q \rightarrow q^{\prime}$ in $\mathcal{A}_{\nu}$ for all $\nu>0$. There exists a path $\pi_{0} \rho_{0} \pi_{1} \rho_{0} \ldots \pi_{n}$ of $\mathcal{A}$ and states $q=q_{0}, q_{0}^{\prime}, q_{1}, q_{1}^{\prime}, \ldots, q_{n}=q^{\prime}$, such that for all $0 \leq$ $i \leq n-1$, and any $\nu>0, q_{i} \stackrel{\pi_{i}}{\longrightarrow} q_{i}^{\prime}$, $q_{i}^{\prime} \in \operatorname{Pre}_{\rho_{i}}^{*}(\top)$, and $q_{i+1} \in \operatorname{Post}_{\left(\rho_{i}\right)_{\nu}}^{*}(\top)$.

Notice that the sequence of states $q_{0}, q_{0}^{\prime}, \ldots$ is independent of $\nu>0$ in the above lemma, and that the enlargement is only used in $\operatorname{Post}_{\left((\rho)_{i}\right)_{\nu}}^{*}(\top)$.

The algorithms of [1320] consist in a usual forward exploration on zones augmented with the application of Lemma 1. by enumerating all cycles in a flat timed automaton. This cannot be extended to general timed automata since the cycles that appear in Lemma 2 are not necessarily simple. This has been a major obstacle against general robust safety algorithms for timed automata.

\section{Infinitesimally Enlarged DBMs}

We define infinitesimally enlarged DBMs (IEDBM), a parameterized extension of DBMs, which we will use to explore the state space of enlarged timed automata. These were first defined in 14 to be used solely as a proof technique. Here, we extend this data structure with additional properties and explicit computations of the bounds on parameter $\delta$, and show how it can be used to efficiently explore the state space.

We fix a clock set $\mathcal{C}_{0}$ including the 0 clock. An infinitesimally enlarged $D B M$ $(I E D B M)$ is a pair $(M, P)_{\left\langle 0, \delta_{0}\right\rangle}$ where $M$ is a DBM and $P$ is a $\left|\mathcal{C}_{0}\right| \times\left|\mathcal{C}_{0}\right|$ matrix over $\mathbb{N}$, called the enlargement matrix. The value $\delta_{0} \in(0, \infty)$ is an upper bound on the unknown parameter $\delta$. Intuitively, an $\operatorname{IEDBM}(M, P)_{\left\langle 0, \delta_{0}\right\rangle}$ represents the set of DBMs $M+\nu P$ where $\nu \in\left[0, \delta_{0}\right)$. Figure 2 shows an example. We often see, abusively, an IEDBM as a matrix over pairs $(m, p) \in \mathbb{Z} \times \mathbb{N}$. The component $(x, y)$ is denoted by $(M, P)_{\left\langle 0, \delta_{0}\right\rangle}[x, y]$. For simplicity, we always consider the half-open intervals of the form $\left[0, \delta_{0}\right)$ even though $\nu$ can be chosen equal to $\delta_{0}$ in some cases. This is not a loss of generality since we are interested in the small values of $\nu$. 
IEDBMs will allow us to reason on the parametric state space of enlarged timed automata "for small values of $\delta$ ", which means that our computations on the data structure will hold for all $\nu \in\left[0, \delta_{0}\right)$, where $\delta_{0}>$ 0 is bound that is possibly updated to a smaller value after each operation. For instance, given sets $Z_{1}=1 \leq x \leq 2+3 \nu$ and $Z_{2}=x \leq 3$, for unknown $\delta$, and assume we want to compute their intersection. We will write $Z_{1} \cap Z_{2}=1 \leq x \leq 2+3 \delta$ and chose $\delta_{0} \leq \frac{1}{3}$. To make these simplifications, we need to compare pairs of IEDBM components in a similar spirit. For instance, to make the above simplification, we write $(2,3)_{\left\langle 0, \frac{1}{3}\right\rangle} \leq(3,0)_{\left\langle 0, \frac{1}{3}\right\rangle}$, which means that $2+3 \nu \leq 3$ for all $\nu \in\left[0, \frac{1}{3}\right)$. We formalize this in the next subsection. To ease reading, we may omit $\delta_{0}$ from IEDBMs if it is clear from the context.

\subsection{Operations on IEDBMs}

We are now going to formalize the simplifications and the operations done on IEDBMs. In order to use our data structure for parametric exploration, we need to define basic operations on timed automata. Similar to DBMs, IEDBMs have reduced forms. To define the reduction operations, we define the + and min operations.

We define the sum as $(m, p)_{\left\langle 0, \delta_{0}\right\rangle}+(n, q)_{\left\langle 0, \delta_{1}\right\rangle}=(m+n, p+q)_{\left\langle 0, \min \left(\delta_{0}, \delta_{1}\right)\right\rangle}$. As an example of this operation, assume that we have the constraints $x-y \leq 2+3 \delta$, and $y \leq 1+\delta$ with the respective upper bounds $\delta_{0}$ and $\delta_{1}$. Then, by summation, we may deduce $x \leq 3+4 \delta$ with the upper bound $\delta_{2}=\min \left(\delta_{0}, \delta_{1}\right)$.

Lemma 3. Given $\left(m_{1}, p_{1}\right)_{\left\langle 0, \delta_{1}\right\rangle},\left(m_{2}, p_{2}\right)_{\left\langle 0, \delta_{2}\right\rangle}$, one can compute $i \in\{1,2\}$, and $\delta_{3}$ such that $\forall \nu \in\left[0, \delta_{3}\right), m_{i}+\nu p_{i}=\min \left(m_{1}+\nu p_{1}, m_{2}+\nu p_{2}\right)$. We denote this by $\left(m_{i}, p_{i}\right)_{\left\langle 0, \delta_{3}\right\rangle}=\min \left(\left(m_{1}, p_{1}\right)_{\left\langle 0, \delta_{1}\right\rangle},\left(m_{2}, p_{2}\right)_{\left\langle 0, \delta_{2}\right\rangle}\right)$.

We write $\left(m_{1}, p_{1}\right)_{\left\langle 0, \delta_{1}\right\rangle} \leq\left(m_{2}, p_{2}\right)_{\left\langle 0, \delta_{2}\right\rangle}$ iff $\min \left(\left(m_{1}, p_{1}\right)_{\left\langle 0, \delta_{1}\right\rangle},\left(m_{2}, p_{2}\right)_{\left\langle 0, \delta_{2}\right\rangle}\right)=$ $\left(m_{1}, p_{1}\right)_{\left\langle 0, \delta_{3}\right\rangle}$ for some $\delta_{3}$; and $\left(m_{1}, p_{1}\right)_{\left\langle 0, \delta_{1}\right\rangle}<\left(m_{2}, p_{2}\right)_{\left\langle 0, \delta_{2}\right\rangle}$ if $m_{1} \neq m_{2} \vee p_{1} \neq p_{2}$.

Intuitively, just like in regular DBMs, we will use the minimum operation to compute conjunctions of constraints. For instance, we already saw above that $x \leq 2+3 \nu \wedge x \leq 3$ is simplified to $x \leq 2+3 \nu$ given $\nu \in\left[0, \frac{1}{3}\right)$. This will be written $\min \left((2,3)_{\langle 0, \infty\rangle},(3,0)_{\langle 0, \infty\rangle}\right)=(2,3)_{\frac{1}{3}}$. It should be clear that for any Boolean combination $\Phi$ of inequalities written between elements of the form $(m, p)_{\left\langle 0, \delta_{1}\right\rangle}$, one can compute, by Lemma [3] a bound $\delta_{0}$ such that either $\Phi[\delta \leftarrow \nu]$ holds for all $\nu \in\left[0, \delta_{0}\right)$, or $\neg \Phi[\delta \leftarrow \nu]$ holds for all $\nu \in\left[0, \delta_{0}\right)$. 
If different upper bounds on $\delta$ are given for two elements to be compared, then we first update the bounds to the minimum of the two bounds. More generally, we assume that all components of an IEDBM have the same bound $\delta_{0}$.

We say that an IEDBM $(M, P)_{\left\langle 0, \delta_{0}\right\rangle}$ is reduced if for all $x, y, z \in \mathcal{C}_{0}$, $(M, P)_{\left\langle 0, \delta_{0}\right\rangle}[x, y] \leq(M, P)_{\left\langle 0, \delta_{0}\right\rangle}[x, z]+(M, P)_{\left\langle 0, \delta_{0}\right\rangle}[z, y]$. IEDBMs can be reduced by the usual Floyd-Warshall algorithm, using the above min and + operations:

Lemma 4. Any $\operatorname{IEDBM}(M, P)_{\left\langle 0, \delta_{0}\right\rangle}$ can be reduced in time $O\left(\left|\mathcal{C}_{0}\right|^{3}\right)$. Moreover, if $(M, P)_{\left\langle 0, \delta_{0}\right\rangle}$ is reduced, then for all $\nu \in\left[0, \delta_{0}\right), M+\nu P$ is a reduced DBM.

When we consider the complexity of minimization as in Lemma 3, we assume that operations on rationals are elementary operations (i.e. they can be performed in constant time). For a more precise analysis, one can incorporate the cost of these computations; for instance, the reduction operation in the previous lemma makes $O\left(\left|\mathcal{C}_{0}\right|^{3}\right)$ minimization operations, so as many operations on rationals.

We define the parametric inclusion by $(M, P)_{\left\langle 0, \delta_{1}\right\rangle} \sqsubseteq(N, Q)_{\left\langle 0, \delta_{2}\right\rangle}$ if, and only if for all $x, y \in \mathcal{C},(M, P)[x, y] \leq(N, Q)[x, y]$.

Lemma 5. One can compute, given $\left(N_{1}, Q_{1}\right)_{\left\langle 0, \delta_{1}\right\rangle},\left(N_{2}, Q_{2}\right)_{\left\langle 0, \delta_{2}\right\rangle}$, and $R \subseteq \mathcal{C}$, and in time $O\left(|\mathcal{C}|^{3}\right)$,

- a reduced IEDBM $(M, P)_{\left\langle 0, \delta_{0}\right\rangle}$, written $\left(N_{1}, Q_{1}\right)_{\left\langle 0, \delta_{1}\right\rangle} \sqcap\left(N_{2}, Q_{2}\right)_{\left\langle 0, \delta_{2}\right\rangle}$, such that $M+\nu P=\left(N_{1}+\nu Q_{1}\right) \cap\left(N_{2}+\nu Q_{2}\right)$ for all $\nu \in\left[0, \delta_{0}\right)$,

- a reduced IEDBM $(M, P)_{\left\langle 0, \delta_{0}\right\rangle}$, written $\operatorname{PReset}_{R}\left(\left(N_{1}, Q_{1}\right)_{\left\langle 0, \delta_{1}\right\rangle}\right)$, such that $M+\nu P=\operatorname{Reset}_{R}\left(N_{1}+\nu Q_{1}\right)$ for all $\nu \in\left[0, \delta_{0}\right)$,

- a reduced IEDBM $(M, P)_{\left\langle 0, \delta_{0}\right\rangle}$, written PPost I $_{\geq 0}\left(\left(N_{1}, Q_{1}\right)_{\left\langle 0, \delta_{2}\right\rangle}\right)$, such that $M+\nu P=$ Post $_{\geq 0}\left(N_{1}+\nu Q_{1}\right)$ for all $\nu \in\left[0, \delta_{0}\right)$.

We are going to define the parametric post operation along an edge $e$. By a slight abuse of notation, we will see any (non-enlarged) guard $g$ as the IEDBM $(g, \mathbf{0})_{\langle 0, \infty\rangle}$. When we consider the enlargement $(g)_{\delta}$ of a guard, this will also refer to the corresponding IEDBM with $\delta_{0}=\infty$. By combining these operations, for a given edge $e$, we define PPost ${ }_{e}\left((M, P)_{\left\langle 0, \delta_{0}\right\rangle}\right)=\operatorname{PPost}_{\geq 0}\left(\operatorname{PReset}_{R}(g \sqcap\right.$ $\left.\left.(M, P)_{\left\langle 0, \delta_{0}\right\rangle}\right)\right)$, where $g$ is the guard of $e$, and $R$ its reset set. By Lemma 5 this corresponds to $\operatorname{Post}_{e}(M+\delta P)$ for sufficiently small $\delta$.

We refer to pairs of locations and IEDBMs as symbolic states. We extend the parametric post operator to symbolic states by $\operatorname{PPost}_{e}((\ell, Z))=\left(\ell^{\prime}, Z^{\prime}\right)$ where $e=\left(\ell, g, R, \ell^{\prime}\right)$, and $Z^{\prime}=\operatorname{PPost}_{e}(Z)$.

Lemma 6. For any sequence of edges $e_{1} \ldots e_{n}$, and symbolic state $(\ell, Z)$, if $\operatorname{PPost}_{\left(e_{1}\right)_{\delta}\left(e_{2}\right)_{\delta} \ldots\left(e_{n-1}\right)_{\delta}}((\ell, Z))=\left(\ell^{\prime}, Z^{\prime}\right)$, and $\left(\ell^{\prime}, Z^{\prime}\right) \neq \emptyset$, then there exists $\delta_{0}>$ 0 such that for all $\nu \in\left[0, \delta_{0}\right)$, and state $q^{\prime} \in\left(\ell^{\prime}, Z^{\prime}\right)[\delta \leftarrow \nu]$, there exist $q \in$ $(\ell, Z)[\delta \leftarrow \nu]$ such that $\left(\ell_{1}, q\right) \stackrel{\left(e_{1}\right)_{\nu} \ldots\left(e_{n-1}\right)_{\nu}}{\longrightarrow}\left(\ell_{n}, q^{\prime}\right)$.

Let the width of $(M, P)_{\left\langle 0, \delta_{0}\right\rangle}$ be defined as $\operatorname{width}(M, P)=\max _{x, y \in \mathcal{C}_{0}} P_{x, y}$.

\subsection{Parametric Abstractions}

We will first recall some abstractions applied on zones in a non-parametric setting, then generalize them to symbolic states described by IEDBMs. 
Closures and LU-abstraction. Let $\alpha: \mathcal{C} \rightarrow \mathbb{N}$ be a bound function, and $\eta$ a granularity. The $\alpha, \eta$-closure of a zone $Z$ is the union of the $\alpha, \eta$-regions which intersects it. It is known that when $\alpha$ denotes the maximal constants to which each clock is compared in a timed automaton $\mathcal{A}$, and $\eta$ the granularity of $\mathcal{A}$, a forward exploration based on $\alpha, \eta$-closures is sound and complete [8]. However because closures are not convex, other abstractions have been in use in practical tools; one of them is $L U$-abstraction, where the idea is to relax some of the facets of a zone taking into consideration the maximal constants that appear in the guards of the timed automaton. We will recall the formal definition of LUabstraction by adapting it to DBMs with only non-strict inequalities by a slight change. The correctness of the abstraction is preserved (proved in Lemma 7).

For a timed automaton $\mathcal{A}$ with granularity $\eta$, we define the two bound functions $L, U: \mathcal{C} \rightarrow \mathbb{N}_{\eta}$, called the $L U$-bounds where $L(x)$ (resp. $U(x)$ ) is the largest constant $c$ such that the constraint $x \geq c$ (resp. $x \leq c$ ) appears in some guard or invariant. Given LU-bounds $L, U$, for any DBM $M$, we define $M^{\prime}=\operatorname{Extra}_{L U}^{+}(M)$ as follows.

$$
M_{x, y}^{\prime}= \begin{cases}\infty & \text { if } M_{x, y}>L(x), \text { or }-M_{0, x}>L(x) \\ \infty & \text { if }-M_{0, y}>U(y), x \neq 0 \\ -U(y)-1 & \text { if }-M_{0, y}>U(y), x=0 \\ M_{x, y} & \text { otherwise. }\end{cases}
$$

We recall the correctness of LU-abstractions and closures for reachability properties. Given LU-bounds $L, U$ we write $\alpha=\max (L, U)$ for the function $\alpha(x)=\max (L(x), U(x))$ for all $x \in \mathcal{C}$.

Lemma 7. For any timed automaton $\mathcal{A}$ with granularity $\eta$, its $L U$-bounds $L, U$, and any path $e_{1} e_{2} \ldots e_{n}$ and extended zone $(\ell, Z)$, define $q_{0}=q_{0}^{\prime}=q_{0}^{\prime \prime}=(\ell, Z)$, and let for $0 \leq i<n, q_{i+1}=\operatorname{Post}_{e_{i}}\left(q_{i}\right), q_{i+1}^{\prime}=\operatorname{Extra}_{L U}^{+}\left(\operatorname{Post}_{e_{i}}\left(q_{i}^{\prime}\right)\right)$, and $q_{i+1}^{\prime \prime}=$ Closure $_{\alpha, \eta}\left(\operatorname{Extra}_{L U}^{+}\left(q_{i}^{\prime \prime}\right)\right)$. Then, $q_{n} \neq \emptyset \Leftrightarrow q_{n}^{\prime} \neq \emptyset \Leftrightarrow q_{n}^{\prime \prime} \neq \emptyset$.

One can thus explore the state space of a timed automaton while systematically applying LU-abstraction at each step. In practice, one does not apply closures since they do not yield convex sets. Nevertheless, a $O\left(|\mathcal{C}|^{2}\right)$-time algorithm was given in [19] to decide whether $M \subseteq$ Closure $_{\alpha}(N)$. Thus, when the regular inclusion test is replaced with the latter one, the exploration becomes equivalent to an exploration using closures [198].

Parametric Closures and LU-abstraction. We would like to use these abstractions in our parametric setting. We will show how these sets can be computed parametrically using IEDBMs. Observe that when we consider parametrically enlarged timed automata, the LU-bounds also depend on $\delta$. Let us denote the parametric $L U$-bounds by $L_{\delta}(x)$ (resp. $U_{\delta}(x)$ ) which is the maximum parametric constant, in the sense of Lemma 3 , which appears in the guards of $\mathcal{A}_{\delta}$ as a lower bound (resp. upper bound) on $x$. We define the parametric $L U$-abstraction, for any IEDBM $(M, P)_{\left\langle 0, \delta_{0}\right\rangle}$ by $\left(M^{\prime}, P^{\prime}\right)_{\left\langle 0, \delta_{1}\right\rangle}=\operatorname{PExtra}_{L_{\delta} U_{\delta}}^{+}\left((M, P)_{\left\langle 0, \delta_{0}\right\rangle}\right)$ obtained 
by applying (11) where $M$ is replaced by $(M, P), L$ and $U$ by $L_{\delta}$ and $U_{\delta}$ respectively. The new upper bound $\delta_{1}$ is computed so that all comparisons in (1) hold.

Lemma 8. Consider any enlarged timed automaton $\mathcal{A}_{\delta}$ and its parametric $L U$ bounds $L_{\delta}, U_{\delta}$. For any $(M, P)_{\left\langle 0, \delta_{0}\right\rangle}$, if we write $\left(M^{\prime}, P^{\prime}\right)_{\left\langle 0, \delta_{1}\right\rangle}=$ PExtra $_{L_{\delta} U_{\delta}}^{+}\left((M, P)_{\left\langle 0, \delta_{0}\right\rangle}\right)$, then for all $\nu \in\left[0, \delta_{1}\right), M^{\prime}+\nu P^{\prime}=\operatorname{Extra}_{L U}^{+}(M+\nu P)$.

Thus, LU-abstractions of enlarged zones have uniform representations for small $\delta$.

For an enlarged timed automaton $\mathcal{A}_{\delta}$ we define $\alpha_{\delta}=\max \left(L_{\delta}, U_{\delta}\right)$. For $\nu>0$, we will denote by $\alpha_{\nu}$ the function obtained from $\alpha_{\delta}$ by instantiating $\delta$ to $\nu$. By a slight abuse of notation, we define the $\alpha_{\nu}$-closure of a zone as its $\left(\alpha_{\nu}, \eta\right)$-closure where $\eta$ is the granularity of $\mathcal{A}_{\nu}$. Here $\mathcal{A}$ will be clear from the context, so there will be no ambiguity. We now adapt the inclusion algorithm of [19] to IEDBMs.

Lemma 9. Given IEDBMs $Z=(M, P)_{\left\langle 0, \delta_{0}\right\rangle}$ and $Z^{\prime}=(N, Q)_{\left\langle 0, \delta_{0}\right\rangle}$, we have $\forall \delta_{0}>0, \exists \nu \in\left[0, \delta_{0}\right), M+\nu P \nsubseteq$ Closure $_{\alpha_{\nu}}(N+\nu Q)$, iff, writing $Z^{\prime}=N+\nu Q$, there exist $x, y \in \mathcal{C}$ such that

1. $\left(Z_{x, 0}^{\prime}<Z_{x, 0}\right.$ and $\left.Z_{x, 0}^{\prime} \leq \alpha_{\delta}(x)\right)$, or $\left(Z_{0, x}^{\prime}<Z_{0, x}\right.$ and $\left.Z_{0, x}+\alpha_{\delta}(x) \geq 0\right)$,

2. or $Z_{0, x}+\alpha_{\delta}(x) \geq 0$, and $Z_{y, x}^{\prime}<Z_{y, x}$, and $Z_{y, x}^{\prime} \leq \alpha_{\delta}(y)+Z_{0, x}$.

Moreover, if this condition doesn't hold, then one can compute $\delta_{1}$ under which we do have the inclusion $M+\nu P \subseteq$ Closure $_{\alpha_{\nu}}(N+\nu Q)$.

Notation 10. We denote the successor operation followed by LU-abstraction as $\operatorname{ExPost}(\cdot)=\operatorname{Extra}_{L U}^{+}(\operatorname{Post}(\cdot))$. For the parametric version, we denote PExPost $(\cdot)$ $=\operatorname{PExtra}_{L_{\delta} U_{\delta}}^{+}(\operatorname{PPost}(\cdot))$, where the bounds $L_{\delta}, U_{\delta}$ are implicit. We furthermore denote by $\sqsubseteq^{c}$ the parametric inclusion check defined by Lemma 9 .

We implicitly assume that when a parametric inclusion check $\coprod^{c}$ is performed, the upper bound $\delta_{0}$ is globally updated to the new bound $\delta_{1}$ given by Lemma 9 ,

\subsection{Parametric Cycle Acceleration}

In [20] a parametric data structure based on the parameterized DBMs of [3] was used to represent the state space under all values of $\delta$ rather than for small values. The corresponding algorithms are based on linear arithmetics of reals. This results in a more complicated data structure which is also more general. IEDBMs simplify this representation by storing the state space only for small values of $\delta$, that is $\delta \in\left[0, \delta_{0}\right)$. To compute cycle accelerations, we recall a result of 20] which bounds the number of iterations to compute pre and post fixpoints of a given cycle.

Lemma $11([\mathbf{2 0}])$. Let $N=|\mathcal{C}|^{2}$. For any cycle $\rho$, if $\operatorname{PPost}_{(\rho)_{\delta}}^{*}(\top) \neq \emptyset$ then $\operatorname{PPost}_{(\rho)_{\delta}}^{*}(\top)=\operatorname{PPost}_{(\rho)_{\delta}}^{N}(\top)$, and if $\operatorname{PPre}_{\rho}^{*}(\top) \neq \emptyset$ then $\operatorname{PPre}_{\rho}^{*}(\top)=\operatorname{Pre}_{\rho}^{N}(\top)$. 
Data: Timed automaton $\mathcal{A}=\left(\mathcal{L}, \operatorname{lnv}, \ell_{0}, \mathcal{C}, E\right)$, and target location $\ell_{T}$.

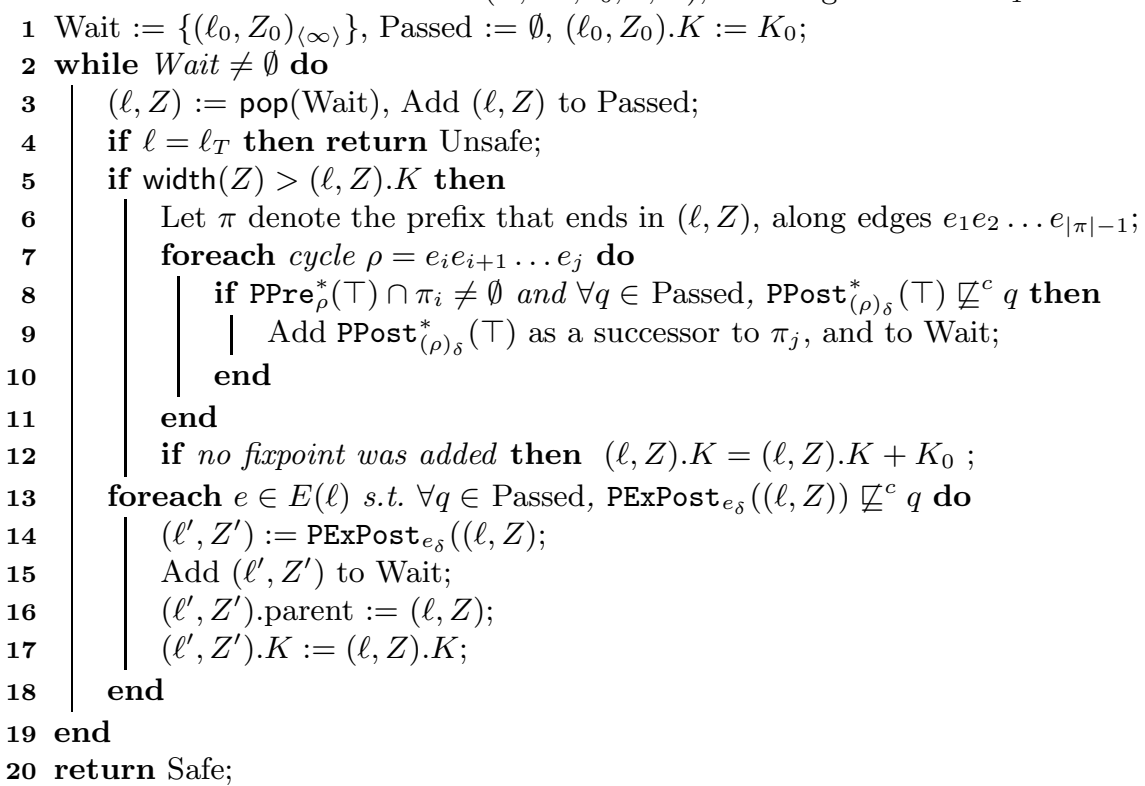

Algorithm 1. Symbolic robust safety semi-algorithm. Here $\left(\ell_{0}, Z_{0}\right)$ is the initial state symbolic state, and $K_{0}$ is a positive constant. We have two containers Wait and Passed storing symbolic states. The search tree is formed by assigning to each visited state $(\ell, Z)$ a parent denoted $(\ell, Z)$.parent (Line [16). We also associate to each symbolic state a bound $K$ on width, denoted $(\ell, Z) . K$.

\section{Symbolic Robust Safety}

Our semi-algorithm consists of a zone-based exploration with IEDBMs using the parametric LU-abstraction and the inclusion algorithm $\sqsubseteq^{c}$ of Lemma 9, It is easy to see that an exploration based on IEDBMs may not terminate in general (see e.g. Fig. 1). Nevertheless, we apply acceleration on well chosen cycles while it is exploring the state space, and it terminated in most of our experiments. To choose the cycles to accelerate, we adopt a lazy approach: we fix a bound $K$, and run the forward search using IEDBMs until the target is reached or some symbolic state has width greater than $K$. In the latter case, we examine the prefix of the current state, and accelerate its cycles by Lemma 1 If no new state is obtained, then we increment the bound $K$ for the current branch and continue the exploration. We thus interpret a large width as the accumulation of imprecisions due to cycles. No cycle may be responsible for a large width, in which case we increase the width threshold and continue the exploration.

We establish the correctness of our semi-algorithm in the following lemma. When it answers Unsafe, it has found a path that ends in the target state, and the proof shows that such a run exists in all $\mathcal{A}_{\nu}$ for $\nu>0$. If it answers Safe, then it has terminated without visiting the target state. If $\delta_{0}$ denotes the upper 
bound on $\delta$ after termination, the proof shows that for all $\nu \in\left[0, \delta_{0}\right)$, an exact exploration applied on $\mathcal{A}_{\nu}$ would visit the same symbolic states as our algorithm when the IEDBMs are instantiated with $\delta \leftarrow \nu$. In other words, the exploration search tree uniformly represents all the search trees that would be generated by an exact algorithm applied on $\mathcal{A}_{\nu}$ for $\nu \in\left[0, \delta_{0}\right)$.

Lemma 12 (Correctness). For any timed automaton $\mathcal{A}$ and location $\ell_{T}$, if Algorithm 1 answers Unsafe then for all $\nu>0, \ell_{T}$ is reachable in $\mathcal{A}_{\nu}$ from the initial state. If it answers Safe, then if $\delta_{0}$ denotes the upper bound on $\delta$ after termination, then for all $\nu \in\left[0, \delta_{0}\right), \mathcal{A}_{\nu}$ does not visit $\ell_{T}$.

\section{Experimental Evaluation}

In this section, we evaluate the performance of our semi-algorithm on several benchmarks from the literature; most of which are available from www.uppaal.org and have been considered in [21, with the exception of the scheduling tests (Sched *) which were constructed from the experiments of [17. We implemented Alg. 11 in OCaml in a tool called Symrob (symbolic robustness, available from www.ulb.ac.be/di/verif/sankur). We consider two other competing algorithms: the first one is the recently published tool Verifix [21] which solves the infinitesimal robust safety problem but does not output any bound on $\delta$. The second algorithm is our implementation of a binary search on the values of $\delta$ which iteratively calls an exact model checker until a given precision is reached.

The exact model checking algorithm is a forward exploration with DBMs using LU extrapolation and the inclusion test of [19] implemented in Symrob. We do not use advanced tricks such as symmetry reduction, federations of zones, and clock decision diagrams; see e.g. 4]. The reason is that our goal here is to compare algorithms rather than software tools. These optimizations could be added to the exact model checker but also to our robust model checker (by adapting to IEDBMs), but we leave this for future work.

In Table 11 the number of visited symbolic states (as IEDBMs for Symrob and as DBMs for Verifix) and the running times are given. On most benchmarks Symrob terminated faster and visited less states. We also note that Symrob actually computed the largest $\delta$ below which safety holds for the benchmarks CSMA/CD and Fischer. One can indeed check that syntactically enlarging the guards by $1 / 3$ (resp. 1/2) makes the respective classes of benchmarks unsafe (Recall that the upper bound $\delta_{0}$ is always strict in IEDBMs). On one benchmark, Verifix wrongly classified the model as non-robust, which could be due to a bug or to the presence of non-progress cycles in the model (see [11]).

Table 2 shows the performance of the binary search for varying precision $\epsilon \in\left\{\frac{1}{10}, \frac{1}{20}, \frac{1}{40}\right\}$. With precision $\frac{1}{10}$, the binary search was sometimes faster than Symrob (e.g. on CSMA/CD), and sometimes slower (e.g. Fischer); moreover, the computed value of $\delta$ was underestimated in some cases (e.g. CSMA/CD and Fischer benchmarks). With precision $\frac{1}{20}$, more precision was obtained on $\delta$ but at a cost of an execution time that is often worse than that of Symrob 
Table 1. Comparison between Symrob (breadth-first search, instantiated with $K_{0}=$ 10) and Verifix [21. The running time of the exact model checking implemented in Symrob is given for reference in the column "Exact" (the specification was satisfied without enlargement in all models). Note that the visited number of states is not always proportional to the running time due to additional operations performed for acceleration in both cases. The experiments were performed on an Intel Xeon 2.67 $\mathrm{GHz}$ machine.

\begin{tabular}{|c|c|c|c|c|c|c|c|}
\hline Benchmark & \multicolumn{2}{|c|}{ Robust $-\delta$} & \multicolumn{2}{|c|}{ Visited States } & \multicolumn{3}{c|}{ Time } \\
\hline & Symrob & Verifix & Symrob & Verifix & Symrob & Verifix & Exact \\
\hline CSMA/CD 9 & Yes $-1 / 3$ & Yes & 147,739 & $1,064,811$ & $61 \mathrm{~s}$ & $294 \mathrm{~s}$ & $42 \mathrm{~s}$ \\
\hline CSMA/CD 10 & Yes $-1 / 3$ & Yes & 398,354 & 846,098 & $202 \mathrm{~s}$ & $276 \mathrm{~s}$ & $87 \mathrm{~s}$ \\
\hline CSMA/CD 11 & Yes $-1 / 3$ & Yes & $1,041,883$ & $2,780,493$ & $12 \mathrm{~m}$ & $26 \mathrm{~m}$ & $5 \mathrm{~m}$ \\
\hline Fischer 7 & Yes $-1 / 2$ & Yes & 35,029 & 81,600 & $11 \mathrm{~s}$ & $12 \mathrm{~s}$ & $6 \mathrm{~s}$ \\
\hline Fischer 8 & Yes $-1 / 2$ & Yes & 150,651 & 348,370 & $45 \mathrm{~s}$ & $240 \mathrm{~s}$ & $24 \mathrm{~s}$ \\
\hline Fischer 9 & Yes -1/2 & Yes & 627,199 & $1,447,313$ & $4 \mathrm{~m}$ & $160 \mathrm{~m}$ & $2 \mathrm{~m} 20 \mathrm{~s}$ \\
\hline MutEx 3 & Yes $-1000 / 11$ & Yes & 37,369 & 984,305 & $3 \mathrm{~s}$ & $131 \mathrm{~s}$ & $3 \mathrm{~s}$ \\
\hline MutEx 4 & No & No & 195,709 & 146,893 & $16 \mathrm{~s}$ & $41 \mathrm{~s}$ & $4 \mathrm{~s}$ \\
\hline MutEx 4 fixed & Yes $-1 / 7$ & - & $5,125,927$ & - & $38 \mathrm{~m}$ & $>24 \mathrm{~h}$ & $7 \mathrm{~m}$ \\
\hline Lip Sync & - & No & - & $29,647,533$ & $>24 \mathrm{~h}$ & $14 \mathrm{~h}$ & $5 \mathrm{~s}$ \\
\hline Sched A & Yes $-1 / 4$ & No* & 9,217 & 16,995 & $11 \mathrm{~s}$ & $248 \mathrm{~s}$ & $2 \mathrm{~s}$ \\
\hline Sched B & No & - & 50,383 & - & $105 \mathrm{~s}$ & $>24 \mathrm{~h}$ & $40 \mathrm{~s}$ \\
\hline Sched C & No & No & 5,075 & 5,356 & $3 \mathrm{~s}$ & $29 \mathrm{~s}$ & $2 \mathrm{~s}$ \\
\hline Sched D & No & No & 15,075 & 928 & $2 \mathrm{~s}$ & $0.5 \mathrm{~s}$ & $0.5 \mathrm{~s}$ \\
\hline Sched E & No & No & 31,566 & 317 & $5 \mathrm{~s}$ & $0.5 \mathrm{~s}$ & $0.5 \mathrm{~s}$ \\
\hline
\end{tabular}

Table 2. Performance of binary search where the initial enlargement is 8 , and the required precision $\epsilon$ is either $1 / 10,1 / 20$ or $1 / 40$. Note that when the model is not robust, the binary search is inconclusive. Nonetheless, in these cases, we do know that the model is unsafe for the smallest $\delta$ for which we model-checked the model. In these experiments the choice of the initial condition (here, $\delta=8$ ) wasn't significant since the first iterations always took negligeable time compared to the case $\delta<1$.

\begin{tabular}{|c|c|c|c|c|c|c|c|}
\hline Benchmark & \multicolumn{2}{|c|}{ Robust $-\delta$} & \multicolumn{2}{|c|}{ Visited States } & \multicolumn{3}{|c|}{ Time } \\
\hline & $\epsilon=1 / 10$ & $\epsilon=1 / 20$ & $\epsilon=1 / 10$ & $\epsilon=1 / 20$ & $\epsilon=1 / 10$ & $\epsilon=1 / 20$ & $\epsilon=1 / 40$ \\
\hline CSMA/CD 9 & Yes $-1 / 4$ & Yes $-5 / 16$ & 151,366 & 301,754 & $43 \mathrm{~s}$ & $85 \mathrm{~s}$ & $123 \mathrm{~s}$ \\
\hline CSMA/CD 10 & Yes $-1 / 4$ & Yes $-5 / 16$ & 399,359 & 797,914 & $142 \mathrm{~s}$ & $290 \mathrm{~s}$ & $428 \mathrm{~s}$ \\
\hline CSMA/CD 11 & Yes $-1 / 4$ & Yes $-5 / 16$ & $1,043,098$ & $2,085,224$ & $8 \mathrm{~m} 20 \mathrm{~s}$ & $17 \mathrm{~m}$ & $26 \mathrm{~m}$ \\
\hline Fischer 7 & Yes $-3 / 8$ & Yes $-7 / 16$ & 75,983 & 111,012 & $15 \mathrm{~s}$ & $21 \mathrm{~s}$ & $31 \mathrm{~s}$ \\
\hline Fischer 8 & Yes $-3 / 8$ & Yes $-7 / 16$ & 311,512 & 462,163 & $53 \mathrm{~s}$ & $80 \mathrm{~s}$ & $129 \mathrm{~s}$ \\
\hline Fischer 9 & Yes $-3 / 8$ & Yes $-7 / 16$ & $1,271,193$ & $1,898,392$ & $5 \mathrm{~m}$ & $7 \mathrm{~m} 30 \mathrm{~s}$ & $12 \mathrm{~m}$ \\
\hline MutEx 3 & Yes -8 & Yes -8 & 37,369 & 37,369 & $2 \mathrm{~s}$ & $2 \mathrm{~s}$ & $2 \mathrm{~s}$ \\
\hline MutEx 4 & \multicolumn{2}{|c|}{ Inconclusive } & $1,369,963$ & $1,565,572$ & $1 \mathrm{~m} 5 \mathrm{~s}$ & $1 \mathrm{~m} 15 \mathrm{~s}$ & $1 \mathrm{~m} 30 \mathrm{~s}$ \\
\hline MutEx 4 fix'd & Yes $-5 / 8$ & Yes $-9 / 16$ & $6,394,419$ & $9,864,904$ & $9 \mathrm{~m} 30 \mathrm{~s}$ & $17 \mathrm{~m}$ & $25 \mathrm{~m}$ \\
\hline Lip Sync & \multicolumn{2}{|c|}{ Inconclusive } & - & - & $>24 \mathrm{~h}$ & $>24 \mathrm{~h}$ & $>24 \mathrm{~h}$ \\
\hline Sched A & \multicolumn{2}{|c|}{\begin{tabular}{|l|l|} 
Yes $-7 / 16$ & Yes $-15 / 32$ \\
\end{tabular}} & 27,820 & 37,101 & $6 \mathrm{~s}$ & $9 \mathrm{~s}$ & $11 \mathrm{~s}$ \\
\hline Sched B & \multicolumn{2}{|c|}{ Inconclusive } & 109,478 & 336,394 & $35 \mathrm{~s}$ & $140 \mathrm{~s}$ & $20 \mathrm{~m}$ \\
\hline Sched C & \multicolumn{2}{|c|}{ Inconclusive } & 10,813 & 36,646 & $2 \mathrm{~s}$ & $6 s$ & $56 \mathrm{~s}$ \\
\hline Sched D & \multicolumn{2}{|c|}{ Inconclusive } & 27,312 & 182,676 & $2 \mathrm{~s}$ & $9 \mathrm{~s}$ & $60 \mathrm{~s}$ \\
\hline Sched E & \multicolumn{2}{|c|}{ Inconclusive } & 98,168 & 358,027 & $6 s$ & $17 \mathrm{~s}$ & $95 \mathrm{~s}$ \\
\hline
\end{tabular}


and systematically more states to visit. Increasing the precision to $\frac{1}{40}$ leads to even longer execution times. On non-robust models, a low precision analysis is often fast, but since the result is inconclusive, one rather increases the precision, leading to high execution times. The binary search can be made complete by choosing the precision exponentially small [11] but this is too costly in practice.

\section{Conclusion}

We presented a symbolic procedure to solve the quantitative robust safety problem for timed automata based on infinitesimally enlarged DBMs. A good performance is obtained thanks to the abstraction operators we lifted to the parametric setting, and to the lazy approach used to accelerate cycles. Although no termination guarantee is given, we were able to treat several case studies from the literature, demonstrating the feasability of robustness verification, and the running time was often comparable to that of exact model checking. Our experiments show that binary search is often fast if run with low precision; however, as precision is increased the gain of a parametric analysis becomes clear. Thus, both approaches might be considered depending on the given model.

An improvement over binary search for a problem of refinement in timed games is reported in 23]; this might be extended to our problem as well. Both our tool and Verifix fail when a large number of cycles needs to be accelerated, and this is difficult to predict. An improvement could be obtained by combining our lazy acceleration technique using the combined computation of the cycles of [21. An extension to LTL objectives could be possible using [9].

\section{References}

1. Altisen, K., Tripakis, S.: Implementation of timed automata: An issue of semantics or modeling? In: Pettersson, P., Yi, W. (eds.) FORMATS 2005. LNCS, vol. 3829, pp. 273-288. Springer, Heidelberg (2005)

2. Alur, R., Dill, D.L.: A theory of timed automata. Theoretical Computer Science 126(2), 183-235 (1994)

3. Annichini, A., Asarin, E., Bouajjani, A.: Symbolic techniques for parametric reasoning about counter and clock systems. In: Emerson, E.A., Sistla, A.P. (eds.) CAV 2000. LNCS, vol. 1855, pp. 419-434. Springer, Heidelberg (2000)

4. Behrmann, G., Bengtsson, J., David, A., Larsen, K.G., Pettersson, P., Yi, W.: Uppaal implementation secrets. In: Damm, W., Olderog, E.-R. (eds.) FTRTFT 2002. LNCS, vol. 2469, pp. 3-22. Springer, Heidelberg (2002)

5. Behrmann, G., Bouyer, P., Larsen, K.G., Pelanek, R.: Lower and upper bounds in zone-based abstractions of timed automata. Int. J. Softw. Tools Technol. Transf. 8(3), 204-215 (2006)

6. Behrmann, G., David, A., Larsen, K.G., Håkansson, J., Pettersson, P., Yi, W., Hendriks, M.: UPPAAL 4.0. In: QEST 2006, pp. 125-126 (2006)

7. Bengtsson, J., Yi, W.: Timed automata: Semantics, algorithms and tools. In: Desel, J., Reisig, W., Rozenberg, G. (eds.) Lectures on Concurrency and Petri Nets. LNCS, vol. 3098, pp. 87-124. Springer, Heidelberg (2004) 
8. Bouyer, P.: Forward analysis of updatable timed automata. Formal Methods in System Design 24(3), 281-320 (2004)

9. Bouyer, P., Markey, N., Reynier, P.-A.: Robust model-checking of linear-time properties in timed automata. In: Correa, J.R., Hevia, A., Kiwi, M. (eds.) LATIN 2006. LNCS, vol. 3887, pp. 238-249. Springer, Heidelberg (2006)

10. Bouyer, P., Markey, N., Reynier, P.-A.: Robust analysis of timed automata via channel machines. In: Amadio, R.M. (ed.) FOSSACS 2008. LNCS, vol. 4962, pp. 157-171. Springer, Heidelberg (2008)

11. Bouyer, P., Markey, N., Sankur, O.: Robust model-checking of timed automata via pumping in channel machines. In: Fahrenberg, U., Tripakis, S. (eds.) FORMATS 2011. LNCS, vol. 6919, pp. 97-112. Springer, Heidelberg (2011)

12. Bozga, M., Graf, S., Mounier, L.: If-2.0: A validation environment for componentbased real-time systems. In: Brinksma, E., Larsen, K.G. (eds.) CAV 2002. LNCS, vol. 2404, pp. 343-348. Springer, Heidelberg (2002)

13. Daws, C., Kordy, P.: Symbolic robustness analysis of timed automata. In: Asarin, E., Bouyer, P. (eds.) FORMATS 2006. LNCS, vol. 4202, pp. 143-155. Springer, Heidelberg (2006)

14. DeWulf, M., Doyen, L., Markey, N., Raskin, J.-F.: Robust safety of timed automata. Formal Methods in System Design 33(1-3), 45-84 (2008)

15. De Wulf, M., Doyen, L., Raskin, J.-F.: Almost ASAP semantics: From timed models to timed implementations. Formal Aspects of Computing 17(3), 319-341 (2005)

16. Dill, D.L.: Timing assumptions and verification of finite-state concurrent systems. In: Sifakis, J. (ed.) CAV 1989. LNCS, vol. 407, pp. 197-212. Springer, Heidelberg (1990)

17. Geeraerts, G., Goossens, J., Lindström, M.: Multiprocessor schedulability of arbitrary-deadline sporadic tasks: Complexity and antichain algorithm. Real-Time Systems. The International Journal of Time-Critical Computing Systems 48(2) (2013)

18. Gupta, V., Henzinger, T.A., Jagadeesan, R.: Robust timed automata. In: Maler, O. (ed.) HART 1997. LNCS, vol. 1201, pp. 331-345. Springer, Heidelberg (1997)

19. Herbreteau, F., Kini, D., Srivathsan, B., Walukiewicz, I.: Using non-convex approximations for efficient analysis of timed automata. In: FSTTCS 2011, pp. 78-89 (2011)

20. Jaubert, R., Reynier, P.-A.: Quantitative robustness analysis of flat timed automata. In: Hofmann, M. (ed.) FOSSACS 2011. LNCS, vol. 6604, pp. 229-244. Springer, Heidelberg (2011)

21. Kordy, P., Langerak, R., Mauw, S., Polderman, J.W.: A symbolic algorithm for the analysis of robust timed automata. In: Jones, C., Pihlajasaari, P., Sun, J. (eds.) FM 2014. LNCS, vol. 8442, pp. 351-366. Springer, Heidelberg (2014)

22. Larsen, K.G., Legay, A., Traonouez, L.-M., Wąsowski, A.: Robust specification of real time components. In: Fahrenberg, U., Tripakis, S. (eds.) FORMATS 2011. LNCS, vol. 6919, pp. 129-144. Springer, Heidelberg (2011)

23. Legay, A., Traonouez, L.-M.: PYECDAR: Towards open source implementation for timed systems. In: Van Hung, D., Ogawa, M. (eds.) ATVA 2013. LNCS, vol. 8172, pp. 460-463. Springer, Heidelberg (2013)

24. Puri, A.: Dynamical properties of timed automata. Discrete Event Dynamic Systems 10(1-2), 87-113 (2000)

25. Swaminathan, M., Franzle, M.: A symbolic decision procedure for robust safety of timed systems. In: TIME 2007, pp. 192-192 (2007)

26. Swaminathan, M., Fränzle, M., Katoen, J.-P.: The surprising robustness of (closed) timed automata against clock-drift. In: Ausiello, G., Karhumäki, J., Mauri, G., Ong, L. (eds.) IFIP TCS 2008. IFIP, vol. 273, pp. 537-553. Springer, Heidelberg (2008) 